\title{
La educación emocional a través de las prácticas motrices de expresión. Perspectiva de género
}

\section{Emotional education through motor expressive activities: A gender perspective}

\author{
Paula Gelpi Fleta* \\ zgzmaster@ecologiaemocional.org \\ Ma Rosario Romero-Martín** \\ rromero@unizar.es \\ Mercé Mateu Serra*** \\ mercemateu@gencat.cat \\ Glòria Rovira Bahillo**** \\ gloriarovira@hotmail.com \\ Pere Lavega Burgués***** \\ plavega@inefc.es \\ * Instituto de Ecología Emocional Zaragoza, Fundación Ambit, España \\ ** Universidad de Zaragoza, España \\ *** Universidad de Barcelona, España \\ **** Universidad de Girona, España \\ ***** Universidad de Lleida
}

\section{Resumen:}

Esta investigación en Educación Física, analiza el tipo de emociones que suscitan las prácticas de expresión motriz (PME) de carácter cooperativo y psicomotor, según la clasificación de Parlebas. La vivencia emocional se analiza además en base al género y a la sede (localización geográfica) donde se desarrolló el estudio.Se aplicó el cuestionario GES (GamesEmotionalscale) sobre conciencia emocional, a 172 estudiantes (61 mujeres y 111 hombres) de primer curso de Ciencias de la Actividad física y el Deporte y de la diplomatura de Maestro en Educación Física de la Universidad de Barcelona y la Universidad de Zaragoza (sedes de Huesca y Zaragoza). El análisis de los datos mediante ecuaciones

\begin{abstract}
:
This study analyses the types of emotions that are aroused by motor expressive activities (MEA) of a cooperative and psychomotor nature, in accordance with the classification of Parlebas. Emotional experience was analysed in relation to gender and the location where the research was conducted. The Games and Emotions Scale (GES), a measure of emotional awareness, was administered to 172 students (61 female and 111 male) recruited from year one of a degree in sports science and from a diploma course for prospective physical education teachers (University of Barcelona and the University of Zaragoza, the two locations). Data analysis by means of generalized estimating equations showed
\end{abstract}


La educación emocional a través de las prácticas motrices de expresión. Perspectiva de género

Paula Gelpi Fleta, Ma Rosario Romero-Martín, Mercé Mateu Serra, Glòria Rovira Bahillo y Pere Lavega Burgués

de estimación generalizadas mostró que las PME activaron en el alumnado valores elevados de emociones positivas. El dominio de cooperación originó emociones positivas más intensas que el psicomotor. No se encontraron diferencias significativas en la intensidad emocional entre hombres y mujeres, lo que contradice la creencia popular y determinada literatura que atribuye a la mujer una mayor emocionalidad. No se encontraron diferencias significativas entre sedes, aunque sí en alguna interacción al relacionar sedes con tipos de emoción y con tipo de dominio. En definitiva esta investigación confirma el papel destacado de las PME para generar experiencias positivas orientadas hacia la promoción del bienestar del alumnado.

\section{Palabras clave:}

Prácticas motrices de expresión; educación física; emociones; género; dominios de acción motriz; cooperación; psicomotor. that MEA evoked strong positive emotions in the students. The cooperative domain evoked more intense positive emotions than did the psychomotor domain. There were no significant differences in the intensity of emotions reported by males and females, a finding that contradicts both popular beliefs and some previous studies that attribute greater emotionality to women. Overall there were no significant differences between the two locations, although some of the interactions were significant when location was analysed in relation to the type of emotion and the type of motor domain. This study confirms the key role that MEA can play in terms of generating positive emotions and promoting wellbeing among students.

\section{Key Words :}

Motor expressive activities; physical education; emotions; gender; motor action domains; cooperation; psychomotor.

\section{Résumé:}

Cette recherche en éducation physique, examine les types d'émotions soulevées des pratiques d'expression motrice (PEM) classés, selon Parlebas, en coopératives et psychomotrices. L'expérience émotionnelle est analysée de plus sur la base du genre et le siège (localisation géographique) où l'étude fut développée. Le questionnaire GES (GamesEmotionalScale) sur la conscience émotionnelle, est appliqué aux 172 étudiants (61 femmes et 111 hommes) de l'Université de Lleida et l'Université de Saragosse (Huesca et Saragosse). L'analyse des données, en utilisant des équations d'estimation généralisées, indique que les PEM activent sur les étudiants des niveaux élevés d'émotions positives. Le domaine de la coopération a causé des émotions positives plus intenses que le domaine psychomotrice. Cependant ne se trouvent pas des différences significatives entre l'intensité émotionnelle exprimée par des hommes et par des femmes, ce qui contredit la croyance populaire et déterminée littérature qu'attribue à la femme une plus grand emocionalité. On n'a pas trouvée des différences attribuables au siège de réalisation; pourtant on a trouvée une certaine interaction en reliant les lieux avec les sortes d'émotions et le type de domaine. En conclusion, cette recherche confirme l'importance du rôle des PME pour générer des expériences positives visant à promouvoir le bien-être des étudiants.

\section{Mots clés:}

Pratiques d'expression motrice; éducation physique; émotions; genre; domaine d'action motrice ; coopération; psychomoteur.

Fecha de recepción: 30-12-2013

Fecha de aceptación: 29-1-2014 
Paula Gelpi Fleta, Ma Rosario Romero-Martín, Mercé Mateu Serra, Glòria Rovira Bahillo y

\section{Fundamentación teórica, antecedentes y planteamiento del problema}

\section{Educación física y educación emocional}

"El alma respira a través del cuerpo, y el sufrimiento, ya empiece en la piel o en la imagen mental, tiene lugar en la carne" (Damasio, A., 1996, p.16).

Es tiempo de acción integrativa en la tarea educativa al aceptar a la persona como un ser multidimensional (Gardner, 1995). Son momentos de nueva lectura de nuestro bagaje cultural y sistema educativo para poder ver qué nos sirve y qué no nos sirve y aprender a vivir de una manera más amplia, completa y satisfactoria, y para resolver problemas de hoy a nivel personal y social (Darder y Bach, 2006).

Es también un momento de inquietud del sector educativo hacia el desarrollo de la inteligencia emocional en las aulas. Toda legislación que evolucione en este siglo deberá abrirse a la educación emocional del sujeto considerándolo de forma explícita y consciente en la planificación pedagógica, utilizándolo para su provecho (Bisquerra, 2008; Gallego y Gallego, 2004; Learreta, 2004; Montávez y Zea, 1998; Obiols, 2005). Las emociones tienen funciones importantes en la persona: motivadora, adaptativa, informativa, evaluativa, social, de crecimiento personal, actúan en procesos mentales, en la toma de decisiones, en el bienestar (Bisquerra, 2003; Damasio, 1996; Plutchik, 1991; Salovey y Mayer, 1990). Todas estas funciones ponen de relieve la importancia de las emociones en nuestras vidas. Según Gallego y Gallego (2004) la adecuada gestión emocional y la armonización entre lo cognitivo y lo emocional deberá contemplarse plenamente como parte del curriculum. Nos proponemos armonizar lo emocional y lo cognitivo con lo corporal y rescatar en un lugar adecuado e importante la práctica motriz en ese proceso de alfabetización emocional (Lavega, March y Filella, 2013; Parlebas, 2001; Pellicer, 2011; Ruano, 2004; Torrents, Mateu, Planas y Dinusôva, 2011). La educación física tiene mucho que aportar en este sentido por su objeto de estudio, ya que considera la persona en sus diferentes dimensiones cuando se manifiesta a través de las conductas motrices (Lagardera y Lavega, 2003; Parlebas, 2001), posibilitando una puerta de entrada directa al mundo de los afectos y de la psique humana. 
La educación emocional a través de las prácticas motrices de expresión. Perspectiva de género

Paula Gelpi Fleta, Ma Rosario Romero-Martín, Mercé Mateu Serra, Glòria Rovira Bahillo y Pere Lavega Burgués

La Praxiología motriz como ciencia de la acción motriz se interesa por identificar la lógica interna de cualquier práctica motriz; es decir, por desvelar las condiciones, modos de funcionamiento, los resultados y la puesta en acción de dichas prácticas motrices (Parlebas, 2001). Cualquier alumno que participe en un juego motor, deporte o situación de expresión motriz deberá adaptarse a las exigencias que impone la lógica interna de esa práctica.

Dada la naturaleza motriz de estas prácticas su intervención se realizará mediante conductas motrices. Este concepto remite a la "organización significante del comportamiento motor" (Parlebas, P., 2001, p. 85). Por ello la conducta motriz corresponde a una intervención estrictamente motriz (saltar o correr) y al mismo tiempo a la activación unitaria de las dimensiones biológica, cognitiva, emocional y social que acompaña a cada respuesta motriz. Se trata de una intervención sistémica, donde el alumnado moviliza todas esas dimensiones simultáneas e interdependientemente, dando testimonio de su personalidad.

Como afirma Parlebas (2001) todas las técnicas corporales, sean las que sean, pueden ser analizadas en términos de conducta motriz. Desde esta perspectiva, ya no es el movimiento lo más importante, sino la persona que se mueve y actúa, sus decisiones motrices, sus impulsos afectivos, su amor al riesgo, sus estrategias corporales, su descodificación motriz etc. La afectividad es la clave de estas conductas motrices (Parlebas, 2001). Los datos afectivos se convierten en partes constitutivas de las mismas y por tanto de todo proceso de aprendizaje de cualquier juego o situación motriz (Lagardera y Lavega, 2003). Por lo tanto, para ayudar a los docentes de educación física a realizar elecciones pedagógicas de forma adecuada sería bueno analizar exhaustivamente las principales clases de situaciones motrices, poner al descubierto la lógica de su funcionamiento y descubrir la influencia que ejercen sobre las conductas motrices de los participantes (Parlebas, 2001).

Cada vez resulta más necesario que los docentes construyan una relación y una acción pedagógica que contribuya a la estructuración positiva de la personalidad y emocionalidad de los educandos (Boix, 2007). Esta es una de las razones fundamentales de nuestro estudio ya que, como educadores, tenemos la posibilidad de proporcionar a nuestro alumnado situaciones motrices que tiendan a generar emociones positivas (Ciarrochi y Scott, 2006; Lagardera y Lavega, 2011; Lavega, Aráujo y Jaqueira, 2013) que contribuyan a la mejora del bienestar 
Paula Gelpi Fleta, Ma Rosario Romero-Martín, Mercé Mateu Serra, Glòria Rovira Bahillo y

personal y social (Bisquerra, 2003; Fernández-Abascal, 2009; Vallés y Vallés, 2007).

Dentro de los programas de educación emocional que actualmente despuntan en España, se presenta el modelo psicopedagógico de Bisquerra (2003) basado en cinco competencias emocionales: conciencia emocional, regulación, autonomía, habilidades sociales y habilidades para la vida. Nuestra investigación gira en torno a la competencia de conciencia emocional (conocer las propias emociones y las emociones de los demás), a través de: la auto-observación, la comprensión de la diferencia de pensamientos, acciones y emociones; la comprensión de las causas de las emociones y la evaluación de la intensidad de las mismas. Comprendemos que las emociones son una pieza clave para el desarrollo inteligente de cualquier otra dimensión del ser humano, ya sea biológica, cognitiva o social.

Entre los distintos tipos de prácticas motrices en este estudio se dirige la atención hacia la expresión motriz.

\section{La expresión motriz}

Las situaciones motrices de expresión (Mateu y Coelho, 2011) se extienden desde aquellas en las que el objetivo es la forma pura de la acción motriz, donde las propias acciones son el mensaje (función poética), hasta situaciones en que la forma remite a un tema, a una referencia externa (función referencial), abarcando un amplio abanico de prácticas que van desde la espontaneidad a la máxima codificación. Se caracterizan por una intencionalidad expresiva-comunicativa (de alteralidad). El objetivo de todas estas prácticas se aleja del pragmatismo de la actividad física centrada en la consecución de habilidades concretas (Romero, 1999; Torrents et al., 2011).

Atendiendo al tipo de interacción motriz entre los participantes, las prácticas motrices de expresión pueden corresponder a dos dominios de acción motriz (Parlebas, 2001): psicomotor (sin interacción entre los protagonistas) y cooperativo (en la que dos o más personas interactúan positivamente para alcanzar un objetivo común).

De la revisión de las investigaciones sobre el estudio de las prácticas de expresión motriz (PEM) y la vivencia emocional destacamos el estudio de Ruano (2004), que tras la elaboración de un cuestionario y un programa de intervención, concluyó que no había mayor vivencia 
La educación emocional a través de las prácticas motrices de expresión. Perspectiva de género

Paula Gelpi Fleta, Ma Rosario Romero-Martín, Mercé Mateu Serra, Glòria Rovira Bahillo y Pere Lavega Burgués

emocional positiva ni mejora del bienestar psicológico del alumnado, si bien apuntaba la necesidad de desarrollar un instrumento de evaluación capaz de medir el impacto de la práctica en el alumnado de una forma que considerara la idiosincrasia de cada sujeto y la particular representación consciente de sus vivencias (Ruano, 2004); la aportación sobre el desarrollo de las habilidades socioemocionales (Learreta, 2004); el estudio del componente emocional y su repercusión en las tareas expresivas (Canales, 2007); proyectos de innovación educativa mediante programas de educación emocional a través de la expresión motriz en primaria (Luesia, Romero-Martín y Gelpi, 2011), en secundaria (Pellicer, 2011) y en el ámbito universitario (Gelpi y Lozano, 2010).

Entre los trabajos que se sirven del marco teórico de la praxiología motriz destacamos el estudio de Torrents et al. (2011) sobre la vivencia de emociones positivas en clases de expresión motriz y danza con alumnos universitarios. Lavega, Lagardera, March, Rovira y Aráujo (en prensa) y Romero-Martín, Gelpi, Mateu y Lavega (2013) también han comparado los efectos de las PEM sobre la vivencia emocional con otro tipo de prácticas (juegos motores e introyección motriz) confirmando el papel extraordinario de las PEM en la activación de emociones positivas.

\section{Género y expresión motriz}

Estudios realizados sobre los efectos de los juegos motores en la vivencia emocional (Lavega, March y Filella, 2013; Lagardera y Lavega, 2011) constatan que es necesario estudiar la variable género para interpretar correctamente los resultados.

La educación física a menudo ha orientado su intervención hacia la promoción de estereotipos de género "tradicionales" (Blández, Fernández y Sierra, 2007; Soler, 2009; Táboas-Pais y Rey-Cao, 2012).

El estudio de los estereotipos de género mediante la actividad física identifica dos grandes conjuntos de rasgos: los instrumentales (identificados con la masculinidad) y los afectivos - expresivos (asociados a los rasgos femeninos) (Blández, et al. 2007; Crawford y Unger, 2004).

Las prácticas de expresión motriz (PME) integran el factor estético y expresivo, por lo que, en nuestro contexto cultural se ha asociado principalmente al género femenino (Alvariñas, Fernández y López, 2009; Ruano, 2004). Si bien este tipo de prácticas tiene cada vez más mayor protagonismo en las clases de educación física, su inclusión 
Paula Gelpi Fleta, Ma Rosario Romero-Martín, Mercé Mateu Serra, Glòria Rovira Bahillo y

ha comportado superar viejos prejuicios sexistas en la educación (Ferez, 2004).

Diferentes estudios constatan que las mujeres son emocionalmente más expresivas que los hombres en la comunicación corporal y verbal; una posible interpretación se explica por tratarse de funciones sociales muy convenientes para el género femenino, como la capacidad de establecer y mantener vínculos, para crear y sostener a la comunidad o para cuidar personas, versus al rol de poder y control atribuidos a los hombres (Candela, Barberá, Ramos y Sarrió, 2001; Feldman, Robin, Pietromonaco y Eyssell, 1998). Sin embargo, en otros estudios se observa que cuando no existe la presión social los autoinformes muestran índices parecidos en ambos géneros (Grossman y Wood, 1993; Feldman, Lane, Sechrest y Schwartz, 2000; Feldman, Robin et al., 1998). Todo esto pone en contraste las creencias ampliamente sostenidas y las investigaciones empíricas, donde las diferencias de género en la emoción sigue sin resolverse, y pone de manifiesto la necesidad de ir más allá del paradigma de las diferencias sexuales en la Inteligencia Emocional y de promover referentes de identidad de género menos estereotipados (Gartzia, Aritzeta, Balluerka y Barbera, 2012).

A partir del marco teórico de referencia este estudio se propuso tres objetivos de investigación:

1. Identificar si las tareas motrices de expresión de carácter psicomotor y cooperativo suscitaban las mismas tendencias de vivencia emocional en el alumnado.

2. Examinar si las tares motrices de expresión originaban intensidades emocionales diferentes en mujeres y hombres.

3. Comprobar si existieron diferencias significativas en la intensidad emocional según la variable contexto geográfico (sede).

\section{Metodología}

\section{Participantes}

Este estudio se realizó con 172 estudiantes (61 mujeres y 111 hombres; rango de edad de 18-31 años; $M=21.58$ años, $D T=6.12$ de dos universidades españolas (3 sedes). Participaron estudiantes de primer curso de Ciencias de la Actividad física y el Deporte o de la diplomatura de Maes- 
La educación emocional a través de las prácticas motrices de expresión. Perspectiva de género

Paula Gelpi Fleta, Ma Rosario Romero-Martín, Mercé Mateu Serra, Glòria Rovira Bahillo y Pere Lavega Burgués

tro en Educación Física. Todos ellos intervinieron voluntariamente. Este estudio fue aprobado por el comité de ética de la Universidad de Lleida.

Tabla 1. Características de los participantes en cada una de las sedes

\begin{tabular}{lccccccccc}
\hline Sedes & Total & mujeres & $\%$ & \multicolumn{3}{c}{ hombres $\%$} & Rango Edad & Media & DT \\
\hline Barcelona & 91 & 22 & 27.20 & 69 & 72.80 & $18-27$ & 20.68 & 3.97 \\
Huesca & 52 & 14 & 26.90 & 38 & 73.10 & $18-27$ & 21.40 & 4.81 \\
Zaragoza & 29 & 25 & 86.20 & 4 & 13.80 & $20-39$ & 27.94 & 6.95 \\
Total & 172 & 61 & 35.67 & 111 & 64.33 & $18-39$ & 21.58 & 6.12 \\
\hline
\end{tabular}

\section{Instrumento}

Para la identificación de la intensidad emocional, se empleó la escala de juegos y emociones GES (Games and Emotion Scale) validada por Lavega, Marchy Filella (2013). Esta escala consistía en una planilla que recogía trece ítems, uno por emoción, (cuatro mociones positivas: alegría, humor, amor y felicidad; seis emociones negativas: ira, rechazo, miedo, ansiedad, vergüenza y tristeza; y tres ambiguas: compasión, sorpresa y esperanza) (Bisquerra, 2003), puntuados con una escala Likert de 0 a 10 (el 0 significaba que no habían sentido esa emoción y el 10 que la habían vivido con la máxima intensidad). El instrumento incluía una breve explicación de cada una de las emociones, a modo de recordatorio, en su parte final.

\section{Procedimiento}

Los participantes realizaron una formación previa consistente en una sesión teórica de hora y media con el objeto de aprender a reconocer las trece emociones y otra práctica de hora y media para conocer el instrumento y experimentar el procedimiento.

El programa constaba de cuatro sesiones de hora y media en las que cada participante realizaba un total de veintidós tareas prácticas, diez de carácter psicomotor y doce de carácter cooperativo. 
Paula Gelpi Fleta, Ma Rosario Romero-Martín, Mercé Mateu Serra, Glòria Rovira Bahillo y

Tabla 2. Ejemplos de las situaciones motrices de expresión (SME)

\begin{tabular}{|c|c|c|c|c|}
\hline INTERVENCIÓN & $\begin{array}{c}\text { Sesión } 1 \\
\text { SME } \\
\text { psicomotrices }\end{array}$ & $\begin{array}{c}\text { Sesión } 2 \\
\text { SME } \\
\text { cooperativas }\end{array}$ & $\begin{array}{c}\text { Sesión } 3 \\
\text { SME } \\
\text { psicomotrices }\end{array}$ & $\begin{array}{c}\text { Sesión } 4 \\
\text { SME } \\
\text { cooperativas }\end{array}$ \\
\hline Sesiones & $\begin{array}{l}\text { La huella de } \\
\text { pintura } \\
\text { Grande-peque- } \\
\text { ño-mediano } \\
\text { Arquitectos } \\
\text { Creación del } \\
\text { árbol (I) } \\
\text { Creación del } \\
\text { árbol (II) }\end{array}$ & $\begin{array}{l}\text { Yo cóncavo y } \\
\text { tú convexo } \\
\text { El árbol colec- } \\
\text { tivo } \\
\text { Hielo y fuego } \\
\text { Juntos pero sí } \\
\text { revueltos } \\
\text { Baile tribal (I) } \\
\text { Baile tribal (II) }\end{array}$ & $\begin{array}{l}\text { Pintando mi } \\
\text { burbuja } \\
\text { Improvisando } \\
\text { Eco-rítmico } \\
\text { El museo (I) } \\
\text { El museo (II) }\end{array}$ & $\begin{array}{l}\text { Máscara a cua- } \\
\text { tro manos } \\
\text { Encajándonos } \\
\text { Máximo y } \\
\text { mínimo } \\
\text { La pareja más } \\
\text { sonora } \\
\text { Música orgáni- } \\
\text { ca (I) } \\
\text { Música orgáni- } \\
\text { ca (II) }\end{array}$ \\
\hline
\end{tabular}

Algunos ejemplos de tareas de Expresión serían: 1. De dominio psicomotor: "Pintando mi burbuja": Movilizar las diferentes partes del cuerpo a partir de la simbolización sobre la temática, pintar su kinesfera, el espacio más próximo que rodea su cuerpo; 2. De dominio cooperativo: "Baile tribal I": Composición coreográfica grupal de una danza en base un ritmo externo de carácter africano, imprimiéndole un sentido simbólico que enriqueciera su carácter expresivo y comunicativo. "Baile tribal II": representación escénica de la coreografía grupal delante de sus compañeros/as.

Al finalizar cada actividad, el alumnado cumplimentó el cuestionario GES en el que anotaron el nivel de intensidad de 0 a 10, que habían experimentado en las trece emociones básicas reseñadas en el documento.

\section{Análisis de los datos}

La prueba de Kolmogorov-Smirnov mostró que los datos eran no paramétricos, ya que seguían una distribución muy asimétrica de las intensidades. Por este motivo se siguió un modelo basado en ecuaciones de estimación generalizadas (GEE) para considerar la correlación entre las puntuaciones del mismo sujeto. Se utilizaron distribuciones de la familia Gausiana, con una estructura de correlación intercambiable.

Se aplicaron comparaciones múltiples post-hoc en todos los factores de más de dos categorías. Se empleó el software estadístico SPSS v.19.0. El modelo consideró dos factores intra-sujetos: tipo de emoción (positiva, 
La educación emocional a través de las prácticas motrices de expresión. Perspectiva de género

Paula Gelpi Fleta, Ma Rosario Romero-Martín, Mercé Mateu Serra, Glòria Rovira Bahillo y Pere Lavega Burgués

negativa y ambigua) y dominio de acción motriz (psicomotor o de cooperación) y dos factores inter-sujetos: género (masculino o femenino) y sede (Barcelona, Huesca, Zaragoza).

\section{Resultados}

El modelo de ecuaciones de estimación generalizadas identificó cuatro interacciones significativas: a) género*emociones; b) dominio*emociones; c) sede*emociones y d) sede*dominio (ver tabla 1), además de encontrar significativas las diferencias en la variable emoción tomada de manera independiente.

Tabla 3. Contrastes de los efectos del modelo

\begin{tabular}{lccc}
\hline & Chi-cuadrado de Wald & gl & Sig. \\
\hline emoción & 760.718 & 2 & $<.001$ \\
dominio & .973 & 1 & .324 \\
género & .107 & 1 & .743 \\
sede & 4.342 & 2 & .114 \\
dominio * emoción & 19.909 & 2 & $<.001$ \\
género * dominio & 2.867 & 1 & .090 \\
género * emoción & 17.334 & 2 & $<.001$ \\
sede * dominio & 7.842 & 2 & .020 \\
sede * género & 3.302 & 1 & .069 \\
sede * emoción & 9.725 & 4 & .045 \\
\hline
\end{tabular}

\section{Tipos de emociones y género}

Se encontraron diferencias significativas en la variable tipo de emoción $(p<.001)$ : las emociones positivas obtuvieron los valores más intensos $(M=4.4)$, las emociones ambiguas registraron valores significativamente inferiores a las positivas $(M=1.88, p<.001)$ y las emociones negativas activaron los valores significativamente más bajos $(M=0.71, p<.001)$. 


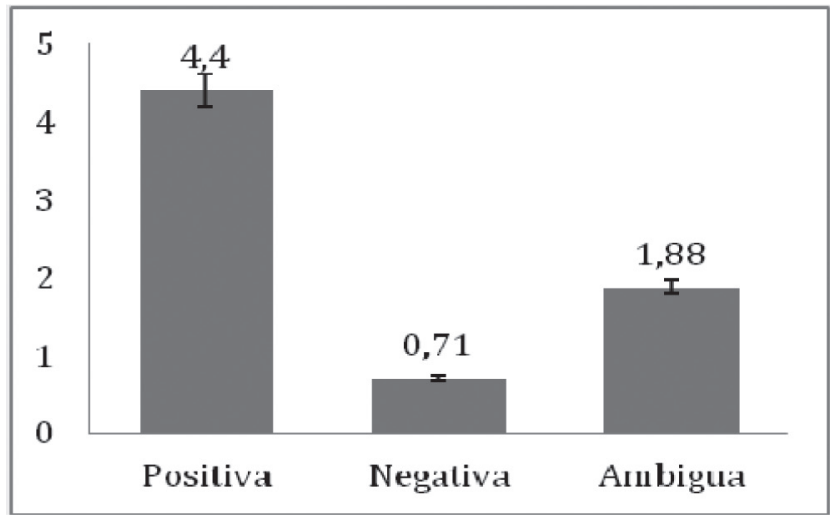

Figura 1. Medias en cada Tipo de emoción

Sin embargo, no se encontraron diferencias significativas entre la intensidad emocional expresada por hombres y por mujeres $(p=.743)$. A pesar de encontrarse diferencias significativas en la interacción género*tipo de emoción $(p<.001)$, la comparación por pares no mostró diferencias entre las intensidades de emociones positivas de hombres y mujeres. No obstante, se observó la tendencia de que las mujeres registraron emociones positivas más intensas $(M=4.52)$ que los hombres $(M=4.22)$. Tampoco se encontraron diferencias entre géneros en las emociones negativas, aunque se observó la tendencia de que las mujeres registraron emociones negativas con valores más bajos $(M=0.63)$ que los hombres $(M=0.82)$.

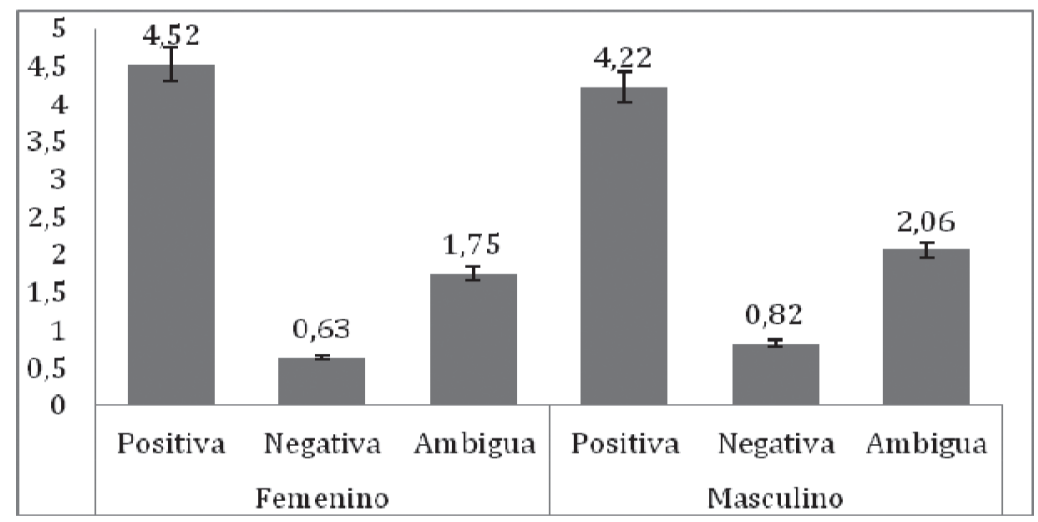

Figura 2. Medias de Género-Tipos de emoción 
La educación emocional a través de las prácticas motrices de expresión. Perspectiva de género

Paula Gelpi Fleta, Ma Rosario Romero-Martín, Mercé Mateu Serra, Glòria Rovira Bahillo y Pere Lavega Burgués

\section{Dominios de acción y tipos de emociones}

No se encontraron diferencias significativas en intensidad emocional entre los dominios de acción psicomotor y cooperativo $(p=.324)$. Sin embargo la interacción dominio*tipo de emoción puso de manifiesto que en las situaciones de cooperación, los valores de las emociones positivas eran más intensos $(M=4.52)$, que en las psicomotrices $(M=4.28 ; p=$ .005). También se observó que el dominio cooperación desencadenaba valores inferiores de emociones negativas $(M=0.62)$ que el dominio psicomotor $(M=0,80, p<.001)$. En las emociones ambiguas el comportamiento fue similar en ambos dominios $(p=.805)$.

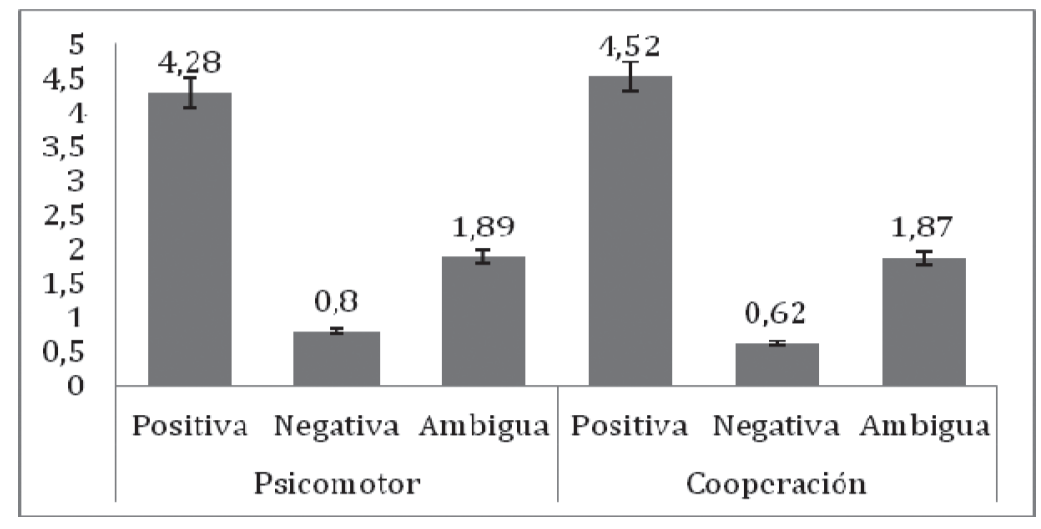

Figura 3. Medias de Dominios de acción-Tipos de emociones

\section{Sede: emociones y dominios}

No se encontraron diferencias significativas entre las tres sedes $(p=$ .114). Las diferencias solo se identificaron en algunas comparaciones de las variables asociadas a la interacción sede*emoción y sede*dominio.

Sede*Emoción: La interacción sede y tipo de emociones mostró diferencias significativas $(p=.045)$. Sin embargo estas diferencias sólo se observaron al comparar las intensidades de emociones positivas entre Barcelona y Huesca. Huesca registró valores significativamente más intensos $(M=4.77)$ que Barcelona $(M=4.09)$. En el resto de casos las sedes se comportaron de manera similar en los tres tipos de emociones. 


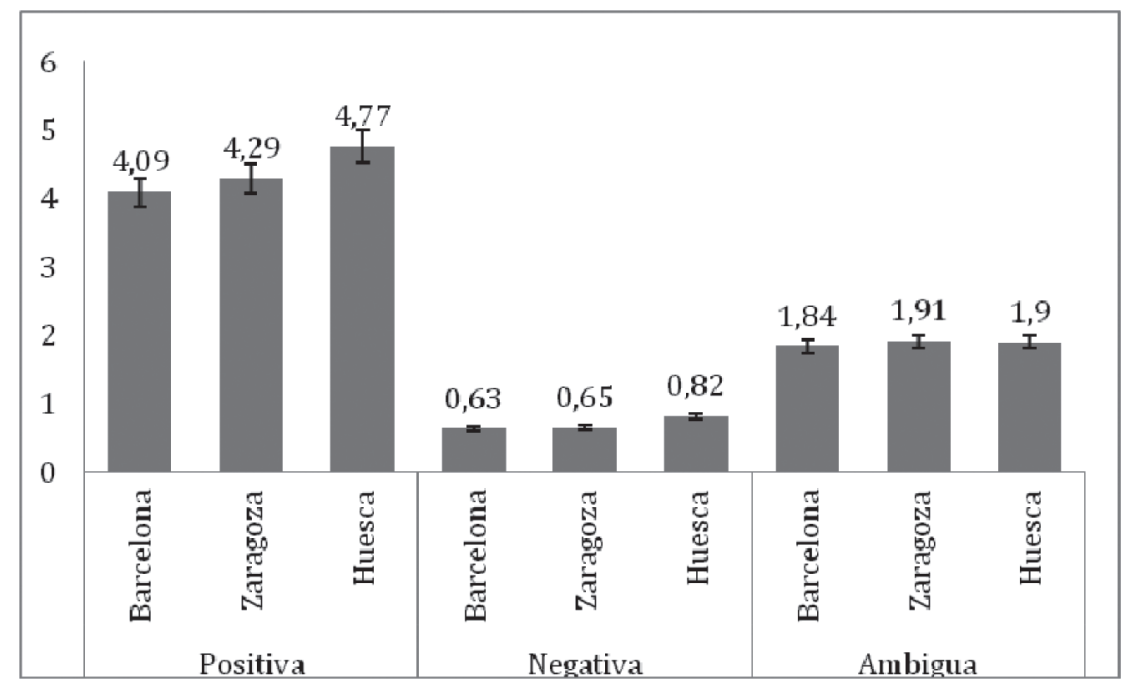

Figura 4. Medias de Sede-Tipos de emociones

Sede*Dominio: Esta interacción mostró diferencias significativas $(p<$ .001). No obstante la única diferencia se encontró entre Huesca y Barcelona en el dominio cooperación. Huesca registró intensidades emocionales superiores $(M=2.52)$ a Barcelona $(M=2.15, p=.013)$. Entre el resto de sedes y dominios no se observaron diferencias significativas.

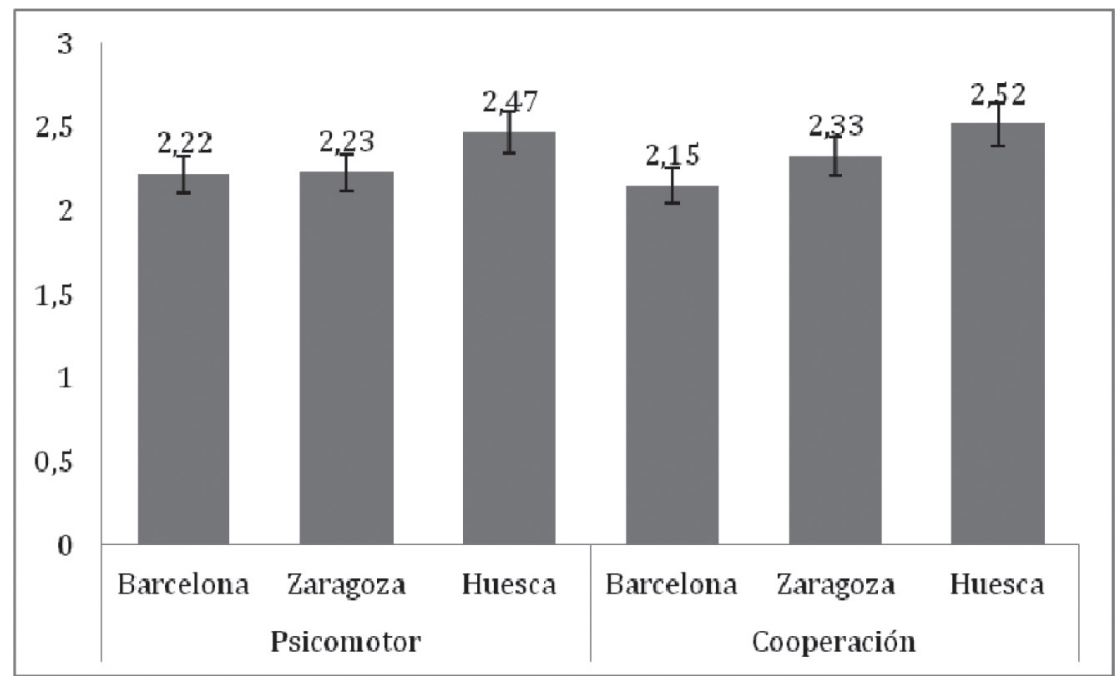

Figura 5. Medias de Dominios de acción-Sede 
La educación emocional a través de las prácticas motrices de expresión. Perspectiva de género

Paula Gelpi Fleta, Ma Rosario Romero-Martín, Mercé Mateu Serra, Glòria Rovira Bahillo y Pere Lavega Burgués

\section{Discusión}

Esta investigación pretendió indagar en la vivencia emocional de estudiantes universitarios en las prácticas de expresión motriz, psicomotrices y de cooperación; además examinó esa vivencia emocional desde la perspectiva de género y contexto geográfico (sede).

Tras el estudio podemos decir, de manera general, que las prácticas de expresión motriz suponen para los participantes una experiencia positiva pudiendo contribuir a su bienestar, como se deriva del hecho de que la intensidad emocional en las emociones positivas es significativamente mayor y de que las emociones negativas originen los valores más bajos. En el estudio de Romero-Martín et al. (2013) se obtenían unos resultados semejantes, es decir, los valores de intensidad más altos se daban en las emociones positivas, que eran superiores al resto, y valores bajos en las emociones negativas; además en ese estudio las prácticas de expresión motriz arrojaban valores más altos en comparación con los juegos populares o con las prácticas de introyección.

Además en todas las interacciones del estudio donde aparece la variable tipo de emoción, y también en el análisis de ese factor de manera independiente, se han dado diferencias significativas. El tipo de emoción, por tanto, es una variable que se ve muy afectada por la puesta en práctica de actividades de expresión motriz.

Todo ello estaría en consonancia con los autores que definen las SME como prácticas en cuya lógica interna tienen un papel fundamental los sentimientos y las emociones (Romero-Martín, 1999, 2001; Ruano, 2004; Torrents y Castañer, 2009; Torrents et al., 2011).

\section{Vivencia emocional y dominios de acción motriz en las prácticas motrices de expresión}

En relación al primer objetivo: "identificar si las tareas motrices de expresión de carácter psicomotor y cooperativo suscitaban las mismas tendencias de vivencia emocional en el alumnado", observamos que el dominio cooperación genera mayor bienestar ya que este tipo de situaciones origina los valores más altos de emociones positivas y los valores más bajos de emociones negativas. Este hallazgo coincide con los resultados de otros estudios realizados mediante juegos deportivos tradicionales (Lagardera y Lavega, 2011; Lavega, Marchy Filella, 2013). 
Paula Gelpi Fleta, Ma Rosario Romero-Martín, Mercé Mateu Serra, Glòria Rovira Bahillo y

Posiblemente la propia lógica interna de las actividades de cooperación, definidas por la necesaria interacción motriz con otros sujetos (Lagardera y Lavega, 2003), provoca un trabajo emocional más intenso ya que el sujeto no sólo pone en juego los aspectos emocionales consigo mismo, sino que está con otros y por tanto debe poner en marcha ajustes de carácter afectivo-relacional. Diferentes estudios muestran la relación de las emociones positivas como el amor/cariño y la alegría con las habilidades sociales (Greenberg y Paivio, 2000; Ruano, 2004).

En el diseño metodológico de las sesiones de expresión motriz existen propuestas donde las actividades cooperativas se suelen utilizar en la primera fase del proceso pedagógico y de la sesión (actividades de introducción) para superar la vergüenza y mejorar el clima grupal y las actividades psicomotrices, por las capacidades de introyección motriz que requiere del alumnado, se proponen estar más presentes en la fase segunda y tercera del proceso de curso, donde las personas ya están más familiarizadas con la disciplina y han alcanzado mayor grado de desinhibición (Montesinos Ayala, 2004; Ruano, 2004).

Por lo tanto, los resultados obtenidos con respecto a la interacción tipo de emoción con el dominio de acción motriz aportan criterios de gran utilidad e interés desde un punto de vista didáctico, como por ejemplo, disponer de criterios para la selección y secuenciación de prácticas motrices de expresión (PME) y para la selección de pautas que acompañen y faciliten la adaptación del alumnado a estas PME.

\section{Vivencia emocional y dominios de acción motriz en las prácticas motrices de expresión}

En relación al segundo objetivo: "examinar si las tareas motrices de expresión originan intensidades emocionales diferentes en mujeres y hom-

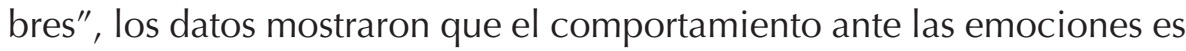
independiente del género.

A diferencia de otros estudios (por ejemplo Alvariñas et al., 2009), que indican que el género femenino se identifica con las actividades físicas de ritmo y expresión, en nuestro estudio, al menos en lo que se refiere a la vivencia emocional expresada, no se ha observado una mayor tendencia de las mujeres respecto de los hombres hacia este tipo de prácticas. Por otro lado, está muy extendida la creencia de que hombres y mujeres se comportan emocionalmente de manera muy diferente 
La educación emocional a través de las prácticas motrices de expresión. Perspectiva de género

Paula Gelpi Fleta, Ma Rosario Romero-Martín, Mercé Mateu Serra, Glòria Rovira Bahillo y Pere Lavega Burgués

(Feldman, Robin et al., 1998). Se ha identificado a las mujeres como el sexo más "emocional", la mujer sensible que se emociona y expresa sus sentimientos con facilidad, aunque a veces se pueda percibir su conducta como irascible o inestable por ello (Sánchez, Fernández-Berrocal, Montañés y Latorre, 2008). Sin embargo las evidencias científicas no muestran resultados concluyentes Ilegando a arrojar datos contradictorios (Alcalá, Camacho, Giner, Giner e Ibáñez, 2006; Sánchez, et al., 2008; Simon y Nath, 2004).

Cómo apuntábamos en el marco teórico, existen estudios que no constatan diferencias entre géneros cuando se comparan las reacciones emocionales en momentos puntuales y cuando la emocionalidad se define como la experiencia de una emoción específica. Esas diferencias se observan cuando las personas estudiadas realizan autoinformes biográficos en los que participa su memoria con sus creencias normativas culturales y de estereotipos de género (Feldman, Lane et al., 2000; Feldman, Robin et al., 1998; Grossman y Wood, 1993). En nuestro estudio, el cuestionario GES se cumplimentaba juego tras juego, de forma inmediata y para cada emoción determinada, por lo que puede venir a corroborar estos estudios.

\section{Vivencia emocional en las prácticas motrices de expresión motriz y contexto geográfico}

Nos planteamos un tercer objetivo: "comprobar si existen diferencias significativas en la intensidad emocional según la variable contexto geográfico (sede)", para el que diferenciamos los estudios de sede*emoción y sede*dominio de acción.

Al estudiar la relación entre las tres sedes con el tipo de emoción y con los dominios de acción motriz, tan sólo se identificaron diferencias significativas entre las sedes de Huesca y Barcelona. En ambos casos la sede de Huesca alcanzó valores más elevados que la de Barcelona, aunque el comportamiento de las emociones y de los dominios fue el mismo en todas las sedes (las emociones positivas y el dominio cooperativo alcanzaron los valores más intensos, mientras que las emociones negativas y el dominio psicomotor registraron los valores más bajos).

A pesar de que se debería ser prudente al avanzar cualquier afirmación concluyente, los resultados parecen confirmar que la fuerza afectiva de la lógica interna de este tipo de prácticas se impone ante 
Paula Gelpi Fleta, Ma Rosario Romero-Martín, Mercé Mateu Serra, Glòria Rovira Bahillo y

otros aspectos externos o contextuales (como localización geográfica, persona que dirigió las sesiones, etc.). Las diferencias geográficas y socioculturales no parecen tener una clara repercusión en los resultados, tal y como se ha observado en otras investigaciones con estudiantes de Coimbra (Portugal), Barcelona y Lleida (España) que mostraron una tendencia similar en la intensidad de las emociones experimentadas, tanto en diferentes dominios de acción motriz (psicomotor y cooperación) como en la clase de emociones (Lavega, Aráujo et al. 2013). Esta tendencia se ha constatado también en investigaciones transnacionales sobre juegos deportivos tradicionales que incluyen centros e investigadores de España, Portugal y Brasil (Lavega, March, Aráujo, Jaqueira, Ribas y Marín, 2011).

\section{Conclusiones}

Las PME, caracterizadas en su lógica interna por el uso, la expresión y la comunicación de las emociones, constituyen una familia de prácticas motrices que desencadena valores intensos de emociones positivas. Por lo tanto, podemos concluir que las actividades de expresión motriz se manifiestan como un recurso educativo que podría favorecer e incrementar el desarrollo del bienestar subjetivo y colectivo de las personas que las realicen. En concreto, aquellas que tienen un carácter cooperativo, se han presentado como movilizadoras de intensidades emocionales positivas más altas comparándolas con las psicomotrices, donde además las emociones negativas se activaron con mayor intensidad.

Nuestro estudio ha revelado que tanto hombres como mujeres muestran una vivencia similar en la intensidad de las distintas emociones (positivas, negativas y ambiguas) en el mismo tipo de práctica, cuestionando los estereotipos de género que han sido atribuidos a las actividades de expresión motriz en el contexto cultural y educativo. Estos resultados invitan a cuestionar las creencias sostenidas ampliamente en nuestra sociedad respecto a las diferencias de respuesta emocional asociada a estereotipos de género y plantean la necesidad de promover intervenciones que garanticen la igualdad de oportunidades fundamentadas en evidencias científicas. Además, se ha observado el papel destacado de la lógica interna de las PME al originar las mismas tendencias en la vivencia emocional del alumnado de los diferentes contextos geográficos. 
La educación emocional a través de las prácticas motrices de expresión. Perspectiva de género

Paula Gelpi Fleta, Ma Rosario Romero-Martín, Mercé Mateu Serra, Glòria Rovira Bahillo y Pere Lavega Burgués

De los hallazgos de esta investigación se derivan implicaciones pedagógicas a la hora de programar las PME y facilitar la adaptación del alumnado a la lógica interna de este tipo de tareas motrices.

Por todo ello, entendemos la expresión motriz como una familia de prácticas motrices que debería tener un papel destacado en el contexto de la educación física. Queda patente la necesidad de adentrarnos en una alfabetización emocional más consciente, planificada, evaluada y con mayor base teórica, para que además pueda repercutir positivamente en el ámbito de enseñanza donde desarrolle su labor profesional nuestro alumnado como futuros/as docentes.

\section{Limitaciones del estudio y prospectiva}

Esta investigación ha estudiado los efectos de las PME sobre la intensidad emocional del alumnado universitario. Entendemos que este estudio debe tener continuidad con el análisis de los datos cualitativos que faciliten profundizar en la comprensión de esa relación entre las PME y la vivencia emocional. La complementación de los datos cuantitativos con los datos cualitativos permitirá comprender mejor el papel de las variables que intervienen tanto desde la lógica interna de estas tareas (dominio de acción motriz) como de las variables externas a estas prácticas (rasgos personales asociados al género, antecedentes deportivos, aspectos motivacionales, etc.).

En otros estudios (Mateu y Coelho, 2011) se ha profundizado en los rasgos de la lógica interna de las PME (además del dominio de acción motriz). Considerando que se conocen esos rasgos que caracterizan la lógica interna para cada PME empleada en este estudio, en investigaciones futuras nos planteamos indagar la vivencia emocional que suscita cada tipo de tarea, pues podría aportar mucha información a la hora de planificar la secuenciación de los contenidos en la asignatura y la selección de pautas didácticas en la facilitación de cada una de ellas.

En esta investigación se han presentado resultados correspondientes a los tres grupos de emociones. También pensamos indagar en estudios posteriores sobre la relación existente entre los diferentes tipos de PME y cada una de las trece emociones estudiadas (cuatro positivas, seis negativas y tres ambiguas). $Y$ nos preguntamos si esta vivencia emocional 
La educación emocional a través de las prácticas motrices de expresión. Perspectiva de género

Paula Gelpi Fleta, Ma Rosario Romero-Martín, Mercé Mateu Serra, Glòria Rovira Bahillo y Pere Lavega Burgués

diferirá cuando los grupos de participantes sean homogéneos (todo varones o todo mujeres) en vez de mixtos como en este estudio.

Finalmente, nos proponemos ahondar en la comprensión de las diferencias encontradas en algunos aspectos emocionales entre las sedes de Huesca y Barcelona. Pensamos que será conveniente relacionar estos datos con los obtenidos mediante alguna otra escala de medida, por ejemplo, de valoración del estado emocional de los participantes antes y después de la actividad, como la escala POMS referida a estados de ánimo (Balaguer, Fuentes, Melià, García-Merita, y Pérez-Recio, 1994), también empleada en otros estudios parecidos (Oiarbide, Martínez-Santos, Usabiaga, Etxebeste y Urdangarín,2014).

\section{Referencias bibliográficas}

Alcalá, V., Camacho, M., Giner, D., Giner, J., \& Ibáñez, E. (2006). Afectos y género. Psicothema, 18(1), 143-148.

Alvariñas, M., Fernández, M. A., \& López, C. (2009). Actividad física y percepciones sobre deporte y género. Revista de Investigación en Educación, 6, 113-122.

Balaguer I., Fuentes I., Melià, J., García-Merita M., \& Pérez-Recio, G. (1994). El perfil de los estados de ánimo (POMS): Baremos para estudiantes valencianos y su aplicación al contexto deportivo. Revista de Psicología del deporte, 1, 35-52.

Bisquerra, R. (2003). Educación emocional y competencias básicas para la vida. Revista de Investigación Educativa (RIE), 21(1),7-43.

Bisquerra, R. (2008). Educación emocional y bienestar. Madrid: Wolters Kluwer Educación.

Blández, J., Fernández-García, E. \& Sierra, M. A. (2007). Estereotipos de género, actividad física y escuela: la perspectiva del alumnado. Revista de Currículum Y Formación Del Profesorado, 2, 1-21.

Boix, C. (2007). Educar para ser feliz. Barcelona: CEAC.

Canales, I. (2007). La mirada y el tacto como condicionantes del compromiso emocional del alumnado en las sesiones de expresión corporal. Motricidad. European Journal of Human Movement, 19, 191-212

Candela, C., Barberá, E., Ramos, A. \& Sarrió, M. (2000). Inteligencia emocional y la variable género. Revista electrónica de Motivación y Emoción, 5(10).

Ciarrochi, J., \& Scott, G. (2006). The link between emotional competence and wellbeing: A longitudinal study. British Journal of Guidance and Counseling, 34, 231-243.

Crawford, M. E., \& Unger, R. K. (2004). Women and Gender. New York: McGraw Hill.

Damasio, A. (1996). El error de Descartes. Barcelona: Crítica.

Darder, P. \& Bach, E. (2006). Aportaciones para repensar la teoría y la práctica educativas desde las emociones. Teoría de la Educación, 18, 55-84. 
La educación emocional a través de las prácticas motrices de expresión. Perspectiva de género

Paula Gelpi Fleta, Ma Rosario Romero-Martín, Mercé Mateu Serra, Glòria Rovira Bahillo y Pere Lavega Burgués

Feldman, L., Robin, L., Pietromonaco, P. R., \& Eyssell, K. M. (1998). Are Women the "More Emotional" Sex? Evidence from emotional experiences in social context.Cognition and Emotion, 12(4), 555- 578.

Feldman, L., Lane, R., Sechrest, L., \& Schwartz, G. (2000). Sex differences in emotional awareness. Personality and Social Psychology Bulletin,26, 1027-1035.

Ferez, S. (2004). De l'expression corporelle aux activités physiques artistiques (APA): Subversion sexuée et différenciation sexuée en éducation physique et sportive (EPS). Staps, 4(66), 113-128.

Fernández-Abascal, E. (Coord.) (2009). Emociones positivas. Pirámide: Madrid.

Gallego, D. J., \& Gallego, M. J. (2004). Educar la inteligencia emocional en el aula. Madrid: PPC.

Gardner, H. (1995). Inteligencias múltiples. La teoría en la práctica. Barcelona: Paidós.

Gartzia, L., Aritzeta, A., Balluerka, N., \& Barbera, E. (2012). Inteligencia emocional y género: más allá de las diferencias sexuales. Anales de Psicología, 28(2), 567-575.

Gelpi, P., \& Lozano, P. (2010). Desarrollo de las competencias emocionales desde el área de Expresión corporal. Las emociones en movimiento en las aulas de la universidad: estudio experimental para el alumnado de $1^{\circ}$ curso de la Facultad de Educación de la Universidad de Zaragoza. Comunicación presentada al V Congreso Internacional y XXVI Congreso Nacional de Educación Física. Barcelona: INEFC.

Gelpi, P., \& Lozano, P. (2010). Las emociones en movimiento en las aulas de la universidad: posibilidades de la expresión corporal en la génesis de emociones positivas y bienestar como medio de educación emocional. Comunicación presentada a las VI Jornadas de Educación Emocional. Barcelona: GROP, Universidad de Barcelona.

Greenberg, L., \& Paivio, S. (2000). Trabajar con la emociones en psicoterapia. Barcelona: Paidós.

Grossman, M., \& Wood, W. (1993). Sex differences in Intensity of emotional experience: A social role interpretation. Journal of Personality and Social Psychology, 65(5), 1010-1022.

Lagardera, F., \& Lavega, P. (2003).Introducción a la praxiología motriz. Barcelona: Paidotribo.

Lagardera, F., \& Lavega, P. (2011). Educación Física, conductas motrices y emociones. Ethologie\& Praxeologie. 16, 23-43.

Lavega, P., Aráujo, P., \& Jaqueira, A. R. (2013). Teaching motor and emotionalcompetencies in universitystudents. Cultura, Ciencia y Deporte, 22(9), 5-15.

Lavega, P., Lagardera, F., March, J., Rovira, G., \& Aráujo P. (en prensa). Efecto de la cooperación motriz en la vivencia emocional positiva: perspectiva de género. Revista Movimento.

Lavega P., March J., Aráujo P., Jaqueira, A. R., Ribas, J. F., \& Marin E. (2011). A CrossCultural experience.Traditional sporting games and emotions in Spain, Portugal and Brazil. En H.D. Guohua Zheng (Ed.), School Physical Education and Students' Quality, Volume XII (pp. 421-425). Liverpool: World Academic Union.

Lavega, P., March, J., \& Filella, G. (2013). Juegos deportivos y emociones. Propiedades psicométricas de la escala GES para ser aplicada en la Educación Física y el Deporte. Revista de investigación educativa, RIE, 31(1), 151-166. 
La educación emocional a través de las prácticas motrices de expresión. Perspectiva de género

Paula Gelpi Fleta, Ma Rosario Romero-Martín, Mercé Mateu Serra, Glòria Rovira Bahillo y Pere Lavega Burgués

Learreta, B. (2004). Los contenidos de expresión corporal en el área de educación física en enseñanza primaria. (Tesis Doctoral inédita). Universidad Complutense de Madrid.

Luesia, L., Romero-Martín, M. R., \& Gelpi, P. (2011). La influencia de la expresión corporal en la educación emocional en alumnos de tercero de educación primaria. Comunicación presentada al I Congreso Internacional: innovaciones en Educación Física. Hacia la inclusión definitiva.Murcia: Asociación de Docentes de Educación Física.

Mateu, M., \& Coelho, M. A. (2011).La lógica interna y los dominios de acción motriz de las situaciones motrices de expresión (SME). Emancipação, 11(1), 129-142.

Montávez, M., \& Zea, M. J. (1998). Expresión corporal: propuestas para la acción. Córdoba: RE-CREA y EDUCA.

Montesinos, D. (2004). La expresión corporal: su enseñanza por el método natural evolutivo. Barcelona: INDE.

Obiols, M. (2005). Diseño, desarrollo y evaluación de un programa de educación emocional en un centro educativo. (Tesis Doctoral inédita). Universidad de Barcelona.

Oiarbide, A., Martínez-Santos, R., Usabiaga, O., Etxebeste, J., \& Urdangarin, C. (2014) Efectos de los juegos de cooperación-oposición en el ánimo de los universitarios. RETOS. Nuevas Tendencias en Educación Física, Deporte y Recreación, 25, 58-62.

Parlebas, P. (2001). Juegos, Deporte y Sociedad. Léxico de Praxiología Motriz. Barcelona: Paidotribo.

Pellicer, I. (2011). Educación física emocional: de la teoría a la práctica. Barcelona: INDE.

Plutchik, R. (1991). The Emotions. Nueva York: University Press of America.

Romero-Martín, M. R. (1999). La expresión y comunicación corporal desde el ámbito de la educación física: elementos, características y enfoque globalizador de la misma. En D. Linares, F. Zurita \& J. A. Iniesta (Coords.), Expresión y comunicación corporal en educación física (pp. 69-99). Granada: Grupo Editorial Universitario.

Romero-Martín, M. R. (2001). Cuerpo y creatividad. Reflexiones sobre la creatividad en educación física. Tándem, 3, 25-38.

Romero-Martín, M. R., Gelpi, P., Mateu, M., \& Lavega, P. (2013). Influencia de juegos y prácticas de expresión e introyección en la vivencia de emociones. Perspectiva de género. Comunicación presentada al Congreso internacional de inteligencia emocional y binestar (CIIE). Zaragoza: Asociación Aragonesa de Psico-pedagogía.

Ruano, M. T. (2004). La influencia de la expresión corporal sobre las emociones: un estudio experimental. (Tesis Doctoral inédita). Universidad Politécnica de Madrid.

Salovey, P., \& Mayer, J. D. (1990). Emotional intelligence. Imagination, Cognition, and Personality, 9, 185-211.

Sánchez, M. T., Fernández-Berrocal, P., Montañés, J., \& Latorre, J. L. (2008). ¿Es la inteligencia emocional una cuestión de género? Socialización de las competencias emocionales en hombres y mujeres y sus implicaciones. Revista Electrónica de Investigación Psicoeducativa, 6(2), 455-474.

Simon, R. W., \& Nath, L. E. (2004). Gender and emotion in the United States: Do men and women differ in self-reports of feelings and expressive behavior? The American Journal of Sociology, 109(5), 1137-1177. 
La educación emocional a través de las prácticas motrices de expresión. Perspectiva de género

Paula Gelpi Fleta, Ma Rosario Romero-Martín, Mercé Mateu Serra, Glòria Rovira Bahillo y Pere Lavega Burgués

Soler, S. (2009). Los procesos de reproducción, resistencia y cambio de las relaciones tradicionales de género en la educación física: el caso del fútbol. Cultura y Educación, 21(1), 31-42.

Táboas-Pais, M. I., \& Rey-Cao, A. (2012). Gender differences in physical education textbooks in Spain: A content analysis of photographs. Sex Roles, 67, 389-402.

Torrents, C., \& Castañer, M. (2009). Las consignas en la expresión corporal: una puerta abierta para la creatividad y la creación coreográfica. Tándem, 30, 111-121.

Torrents, C., Mateu, M., Planas, A., \& Dinusôva, M. (2011). Posibilidades de las tareas de expresión corporal para suscitar emociones en el alumnado. Revista de Psicología del Deporte, 20(2), 401-412.

Vallés, A., \& Vallés, C. (2007). Inteligencia emocional. Aplicaciones educativas. Madrid: EOS.

\section{Agradecimientos}

Estos estudios han recibido financiación del Ministerio de Economía y Competitividad, Secretaría de Estado de Investigación, Desarrollo e Innovación de España a través del proyecto I+D+i Juegos Deportivos Psicomotores y de Cooperación DEP2010-21626-C03-01. 